\title{
Placing direct limits on the mass of earth-bound dark matter
}

\author{
Stephen L. Adlei* \\ Institute for Advanced Study, Einstein Drive, Princeton, NJ 08540, USA.
}

\begin{abstract}
We point out that by comparing the total mass (in gravitational units) of the earthmoon system, as determined by lunar laser ranging, with the sum of the lunar mass as independently determined by its gravitational action on satellites or asteroids, and the earth mass, as determined by the LAGEOS geodetic survey satellite, one can get a direct measure of the mass of earth-bound dark matter lying between the radius of the moon's orbit and the geodetic satellite orbit. Current data show that the mass of such earth-bound dark matter must be less than $4 \times 10^{-9}$ of the earth's mass.
\end{abstract}

Current interest in dark matter has been heightened by the recent report by the DAMA/LIBRA collaboration [1] of evidence for galactic halo dark matter, based on their observation of an annual modulation signal. Astrophysical arguments suggest that the galactic halo dark matter mass density is around $0.3\left(\mathrm{GeV} / \mathrm{c}^{2}\right) \mathrm{cm}^{-3}$, but it is still an open question whether in addition to dark matter bound to the galaxy, there may be larger dark matter concentrations bound to the sun, and bound to the earth. The possibility of sun-bound dark matter was discussed in an article of Frère, Ling and Vertongen [2], who pointed out that local dark matter concentrations in the galaxy may have played a role in the formation of the solar system. Their paper, and the papers of Sereno and Jetzer [3], of Iorio [4], and of Khriplovich and Pitjeva [5], use arguments based on planetary orbits to place a limit on a local excess of sun-bound dark matter of order $3 \times 10^{5}$ times the galactic halo dark matter mass density. It is also possible that there may be further local concentrations of earth-bound dark matter, which if large enough could be relevant [6] for understanding the recently reported [7] spacecraft flyby anomaly. Thus, it would be useful to have a direct method for measuring, or at least placing limits on, the mass of earth-bound dark matter.

The aim of this note is to show that it is possible to set a direct limit on the total earth-bound dark matter mass lying between the radius of the moon's orbit and the radius of low lying satellite orbits, such as that of the accurately monitored [8] LAGEOS satellite. For a satellite of negligible mass in a circular orbit around an astronomical body of mass $M$, measurement of the orbit radius $R$ and the orbital period $T$ gives the product $G M$ (with $G$ the Newton gravitation constant) by

${ }^{*}$ Electronic address: adler@ias.edu 
use of the formula

$$
G M=\frac{4 \pi^{2} R^{3}}{T^{2}}
$$

Thus, from a measurement of the radius and period of the LAGEOS orbit, one gets $G M_{\oplus}$, where we have defined the earth mass $M_{\oplus}$ here to include the mass of all earth-bound dark matter lying within the radius of the LAGEOS orbit. Similarly, by a measurement of the orbit and period of lunar orbiters close to the moon [9], one gets $G M_{m}$, where here we have defined the lunar mass $M_{m}$ to include the mass of all moon-bound dark matter lying within the lunar orbiter radius; we shall assume this moon-bound dark matter mass to be negligible for purposes of this analysis. An alternative way of independently determining the moon's mass is to study the orbit of a near-passing asteroid, such as Eros [10], which is influenced by the gravitational field of the moon as well as the earth. From such an analysis one can extract an accurate figure for the ratio $R_{\oplus / m} \equiv\left(G M_{\oplus}+G \Delta M_{\oplus}\right) /\left(G M_{m}+G \Delta M_{m}\right)$, with $\Delta M_{\oplus}$ and $\Delta M_{m}$ denoting respectively possible small contributions from earth-bound and moon-bound dark matter. Again assuming that moon-bound dark matter can be neglected, and expanding in the small quantity $G \Delta M_{\oplus}$, this ratio becomes

$$
R_{\oplus / m}=\frac{G M_{\oplus}}{G M_{m}}(1+\delta)
$$

with the small positive correction $\delta$ given by

$$
\delta=\frac{\Delta M_{\oplus}}{M_{\oplus}}
$$

Finally, let us consider the orbital system comprising the earth and the moon, for which the combined dynamics of the earth-moon system has to be taken into account. ${ }^{1}$ This shows [12] that measuring the relative earth moon distance by lunar laser ranging, together with the moon's orbital period, gives a determination of the combined mass in gravitational units of the earth-moon system, which is $G M_{\text {combined }}=G M_{\oplus}+G M_{m}+G M_{\mathrm{dm}}$, with $M_{\mathrm{dm}}$ now the mass of earth-bound dark matter ${ }^{2}$ lying between the radius of the moon's orbit and the radius of the LAGEOS satellite

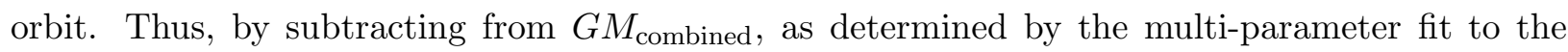

\footnotetext{
${ }^{1}$ The influence of dark matter on the earth-moon system has been investigated earlier with the aim of determining whether the gravitational interaction of galactic dark matter with the ordinary matter in the earth and moon obeys the equivalence principle, and the equivalence principle in this context has been verified to high accuracy; see [1] for details. The assumption that dark matter has normal gravitational interactions with ordinary matter is implicit in our analysis.

${ }^{2}$ If the earth-bound dark matter distribution is not spherically symmetric, then there will be dark-matter contributions to higher multipoles of the earth's gravitational potential, as well as to the monopole mass.
} 
lunar laser ranging experiment, the values of $G M_{\oplus}$ determined by LAGEOS and $G M_{m}$ determined by the lunar orbiters, one can get a direct determination of $G M_{\mathrm{dm}}$,

$$
G M_{\mathrm{dm}}=G M_{\text {combined }}-G M_{\oplus}-G M_{m}
$$

subject to our assumption that the moon-bound dark matter lying within the lunar orbiter radius can be neglected. Alternatively, if one uses an asteroid determination of $G M_{m}$, the subtraction to be performed is

$$
G M_{\text {combined }}-G M_{\oplus}-\frac{G M_{\oplus}}{R_{\oplus / m}} \simeq G M_{\mathrm{dm}}+G M_{m} \delta=G M_{\mathrm{dm}}+\frac{M_{m}}{M_{\oplus}} G \Delta M_{\oplus}
$$

Since $M_{m} / M_{\oplus} \simeq 0.0123$, (15) gives

$$
G M_{\text {combined }}-G M_{\oplus}-\frac{G M_{\oplus}}{R_{\oplus / m}} \simeq G M_{\mathrm{dm}}+0.0123 G \Delta M_{\oplus}>G M_{\mathrm{dm}}
$$

Moreover, if one assumes the earth-bound dark matter $\Delta M_{\oplus}$ relevant for the asteroid orbit to be similar in magnitude to the earth-bound dark $M_{\mathrm{dm}}$ matter lying between the moon's orbit and the LAGEOS orbit, then (6) becomes

$$
G M_{\text {combined }}-G M_{\oplus}-\frac{G M_{\oplus}}{R_{\oplus / m}} \simeq G M_{\mathrm{dm}}[1+O(.01)]
$$

giving a determination of $M_{\mathrm{dm}}$ with a potential one percent accuracy, if the quantities on the left hand side of (7) were known to sufficient accuracy. In fact, with current data, the errors in the left-hand side are more significant than the error arising from the unknown term of order 0.01 on the right of (7).

Proceeding now to a numerical evaluation, ${ }^{3}$ the best evaluation of $G M_{\oplus}$ from LAGEOS data is 13] $G M_{\oplus}=398600.4415 \pm 0.0008 \mathrm{~km}^{3} \mathrm{~s}^{-2}$, which when converted to a TDB compatible (Barycentric Dynamical Time compatible) figure is 14] $G M_{\oplus}=398600.4356 \pm 0.0008 \mathrm{~km}^{3} \mathrm{~s}^{-2}$. For $G M_{\text {combined }}$, the lunar ranging fit EP0 in table 1 of [15], in which the sun/(earth + moon) mass ratio was treated as a solution parameter, gives $M_{\odot} / M_{\text {combined }}=328900.5596 \pm 0.0011$, which converts [14] (using $\left.G M_{\odot}=1.32712440018(8) \times 10^{11} \mathrm{~km}^{3} \mathrm{~s}^{-2}\right)$ to $G M_{\text {combined }}=403503.2357 \pm 0.0014 \mathrm{~km}^{3} \mathrm{~s}^{-2}$. The lunar orbiter measurements reported in [9] give $G M_{m}=4902.84 \mathrm{~km}^{3} \mathrm{~s}^{-2}$, with an uncertainty of around $\pm .05 \mathrm{~km}^{3} \mathrm{~s}^{-2}$ based on a comparison with alternative determinations, but a much more accurate value is obtained from the Eros ranging data of [10], which gives $R_{\oplus / m}=81.300570 \pm 0.000005$,

\footnotetext{
${ }^{3}$ The cited papers do not give details about how errors are calculated, but the context suggests that they are estimated errors based on residuals to multi-parameter model fits.
} 
which corresponds, using the LAGEOS value for $G M_{\oplus}$, to $G M_{m}=4902.8000 \pm 0.0003 \mathrm{~km}^{3} \mathrm{~s}^{-2}$. Substituting these numbers into (4) or (7) gives

$$
\begin{aligned}
G M_{\mathrm{dm}} & \simeq(403503.2357 \pm 0.0014-398600.4356 \pm 0.0008-4902.8000 \pm 0.0003) \mathrm{km}^{3} \mathrm{~s}^{-2} \\
& =(0.0001 \pm 0.0016) \mathrm{km}^{3} \mathrm{~s}^{-2}=(0.3 \pm 4) \times 10^{-9} G M_{\oplus}
\end{aligned}
$$

with the dominant contribution to the error coming from the error in $M_{\text {combined }}$ from the lunar laser ranging fit. Thus, current data show that the mass of earth-bound dark matter lying between the moon's orbit radius $\sim 384,000 \mathrm{~km}$ and the LAGEOS orbit radius ${ }^{4} \sim 12,300 \mathrm{~km}$ must be less than $4 \times 10^{-9}$ of the earth's mass, at a $1 \sigma$ confidence level. ${ }^{5}$ As the accuracy of lunar laser ranging improves, one can expect this limit on $M_{\mathrm{dm}}$ to improve.

I wish to thank Slava Turyshev for inviting me to speak at the workshop From Quantum to Cosmos - III, Airlie, VA that he organized, and for a subsequent email giving me the numbers and references used in the numerical evaluation of the preceding paragraph. I also wish to thank Peter Bender for a helpful conversation at the Airlie workshop, which was the impetus for this investigation, and J. M. Frère for email correspondence. This work was supported in part by the Department of Energy under grant no. DE-FG02-90ER40542, and I also wish to acknowledge the hospitality of the Aspen Center for Physics.

Added Note Gary Gibbons [16] has pointed out that if one assumes that there is no dark matter bound to the earth, then the comparison of $G M_{\oplus}$ as determined by LAGEOS, with that determined by lunar ranging, gives a bound on possible non-Newtonian modifications to the gravitational force, and he has alerted me to several references [17], [18], [19] where the use of satellite orbits to restrict non-Newtonian force models has been discussed. To illustrate with the numbers employed above in the dark matter discussion, if one assumes $G=G_{\text {far }}$ for the $G$ value relevant both for lunar ranging and for the asteroid determination of the earth to moon mass ratio, and $G=G_{\text {near }}$ for the $G$ value relevant for the LAGEOS orbit, and takes $M_{\mathrm{dm}}=0$, then one has

\footnotetext{
${ }^{4}$ The LAGEOS orbit is usually described in terms of its altitude of 5,900 km above the earth's surface, which lies about $6,400 \mathrm{~km}$ from the earth's center.

${ }^{5}$ If dark matter gravitationally bound to the earth were assumed uniformly distributed between the moon's orbit and the LAGEOS orbit, this bound, if saturated, would correspond to a dark matter density of order $10^{10}\left(\mathrm{GeV} / \mathrm{c}^{2}\right) \mathrm{cm}^{-3}$, much higher than the galactic halo density or current limits on the density of dark matter gravitationally bound to the solar system. Just based on this, however, one cannot make any statements on what should have been seen in dark matter detection experiments, since that would require making assumptions about the dark matter density profile around the earth, its mass, and its interaction cross section with ordinary matter, all of which enter into determining the experimental sensitivity. No such model-dependent assumptions enter the purely gravitational analysis given above.
} 
$G_{\text {near }} M_{\oplus}=398600.4356 \pm 0.0008 \mathrm{~km}^{3} \mathrm{~s}^{-2}, G_{\text {far }}\left(M_{\oplus}+M_{m}\right)=403503.2357 \pm 0.0014 \mathrm{~km}^{3} \mathrm{~s}^{-2}$, and $R_{\oplus / m}=M_{\oplus} / M_{m}=81.300570 \pm 0.000005$. When combined these give

$$
\left(G_{\text {near }}-G_{\text {far }}\right) / G=(0.2 \pm 4) \times 10^{-9}
$$

indicating that $G$ can change by at most $\sim 4 \times 10^{-9}$ (the same fractional error that appears in (8) ) between the radius of the LAGEOS orbit and the radius of the moon's orbit. This is a factor of five better than the result given given some time ago by Rapp [18]. However, Turyshev [20], in reviewing fits to lunar ranging, which model the earth-moon distance to $4 \mathrm{~mm}$ accuracy, notes that "analysis of the LLR data tests the gravitational inverse-square law to $3 \times 10^{-11}$ of the gravitational field strength on scales of the Earth-moon distance".

[1] R. Bernabei, P. Belli, F. Cappella, R. Cerulli, C. J. Dai, A. d'Angelo, H. L. He, A. Incicchitti, H. H. Kuang, J. M. Ma, F. Montecchia, F. Nozzoli, D. Prosperi, X. D. Sheng, and Z. P. Ye, "First results from DAMA/LIBRA and the combined results with DAMA/NaI", arXiv:astro-ph/0804.2741.

[2] J.-M. Frère, F.-S. Ling, and G. Vertongen, Phys. Rev. D 77, 083005 (2008).

[3] M. Sereno and Ph. Jetzer, Mon. Not. R. Astron. Soc. 371, 626 (2006).

[4] L. Iorio, JCAP 0605, 002 (2006).

[5] I. B. Khriplovich and E. V. Pitjeva, Int. J. Mod. Phys. D 15, 615 (2006); I. B. Khriplovich, Int. J. Mod. Phys. D 16, 1475 (2007).

[6] S. L. Adler, "Can the flyby anomaly be attributed to earth-bound dark matter?", arXiv:0805.2895.

[7] J. D. Anderson, J. K. Campell, J. E. Ekelund, J. Ellis, and J. F. Jordan, Phys. Rev. Lett. 100, 091102 (2008).

[8] D. P. Rubincam, J. Geophys. Res. 95, 4881 (1990); R. Scharroo, K. F. Wakker, B. A. C. Ambrosius, and R. Noomen, J. Geophys. Res. 96, 729 (1991).

[9] W. H. Michael and W. T. Blackshear, "Recent Results on the Mass, Gravitational Field and Moments of Inertia of the Moon", in The Moon 3, D. Reidel, Dordrecht, pp. 388-402 (1972); available from the NASA Astrophysics Data System.

[10] A. S. Konopliv, J. K. Miller, W. M. Owen, D. K. Yeomans, and J. D. Giorgini, Icarus 160, 289 (2002).

[11] K. L. Nordtvedt, Astrophys. Journ. 437, 529 (1994); K. L. Nordtvedt, J. Müller, and M. Soffel, Astron. Astrophys. 293, L73 (1995).

[12] J. Müller, J. G. Williams, and S. G. Turyshev, "Lunar Laser Ranging Contributions to Relativity and Geodesy", arXiv:gr-qc/0509114.

[13] J. C. Ries, R. J. Eanes, C. K. Shum, and M. M. Watkins, Geophys. Res. Lett. 19, 529 (1992).

[14] S. G. Turyshev, Jet Propulsion Laboratory, private email communication. The quoted value of $G M_{\odot}$ comes from the NASA/JPL website: http://ssd.jpl.nasa.gov/?constants 
[15] J. G. Williams, S. G. Turyshev, and D. H. Boggs, "Lunar Laser Ranging Tests of the Equivalence Principle with the Earth and Moon", arXiv:gr-qc/0507083.

[16] G. W. Gibbons, private email communication.

[17] G. W. Gibbons and B. F. Whiting, Nature Lett. 291, 636 (1981).

[18] R. H. Rapp, Geophys. Res. Lett. 14, 730 (1987).

[19] B. Hubler, A. Cornaz, and W. Kündig, Phys. Rev. D 51, 4005 1995).

[20] S. G. Turyshev, "Experimental Tests of General Relativity", arXiv:0806.1731. 